\title{
The diagnosis and management of antibiotic allergy in children: Systematic review to inform a contemporary approach
}

\author{
Tom Marrs, ${ }^{1,2}$ Adam T Fox, ${ }^{1,2}$ Gideon Lack, ${ }^{1,2}$ George du Toit ${ }^{1,2}$
}

\begin{abstract}
- Additional material is published online only. To view please visit the journal online (http://dx.doi.org/10.1136/ archdischild-2014-306280).

${ }^{1}$ Division of Asthma, Allergy and Lung Biology, Department of Paediatric Allergy, King's College London, London, UK ${ }^{2}$ Department of Paediatric Allergy, Guys and St Thomas' Hospitals NHS Foundation Trust, London, UK
\end{abstract}

\section{Correspondence to} Dr George du Toit, Division of Asthma, Allergy and Lung Biology, Department of Paediatric Allergy, King's College London at St Thomas' Hospital, Westminster Bridge Road, London SE1 7EH, UK; george.dutoit@kcl.ac.uk

Received 23 September 2014 Accepted 26 November 2014 Published Online First 19 December 2014

\section{CrossMark}

\begin{abstract}
Background Adverse drug reactions (ADRs) to antibiotics are commonly reported among children, with some representing genuine drug allergies. Accurate diagnostic tests are required. Drug provocation testing (DPT) is accepted as the gold standard investigation among children with suspected antibiotic allergy. We conducted this review to ascertain the strength of current evidence for using DPT as the first-line investigation for suspected antibiotic allergy among children.
\end{abstract}

Methods Medline was searched in June 2014 for publications investigating antibiotic allergy among children. Results 865 publications were retrieved and 76 studies selected. ADRs are most common among children of $0-4$ years, however only some reveal drug allergies. The best evidence demonstrates that around $0.21 \%$ of general paediatric outpatients demonstrate positive antibiotic intradermal (ID) testing or DPTs, while $6.8 \%$ of children attending emergency departments for suspected $\beta$-lactam allergy may fulfil DPT reactions. Four studies used DPTbased protocols to investigate suspected antibiotic allergy, with two of these conducting ID testing and DPTs across all participants. $\beta$-lactam and clarithromycin ID testing had sensitivities of $66.7 \%$ and $75 \%$, with positive predictive values of $36 \%$ and $33 \%$, respectively, when compared with DPT data.

Conclusions Our literature review found four (6\%) publications that performed DPTs to subjects' index antibiotic across all participants. No rigorous evidence supports using skin prick, ID or in vitro diagnostic testing; indeed, the testing regimens, extracts and positivity criteria used are inconsistent. We recommend that suspected nonserious antibiotic allergy should be primarily investigated using DPT-based clinical protocols. Data examining their safety, acceptability and diagnostic performance are required.

\section{INTRODUCTION}

Adverse drug reactions (ADRs) to antibiotics are commonly reported among children and young people. Allergic mechanisms are frequently suspected and alternative agents routinely prescribed. Altered antibiotic choices may impact on the health of both the individual and wider society, where antibiotic resistance and increasing health costs are becoming more burdensome. ${ }^{1}$

A substantial proportion of children develop rashes, urticaria, angio-oedema and respiratory symptoms while unwell, frequently while taking antibiotics. ${ }^{2}$ Thus many children are diagnosed with 'suspected antibiotic allergy'. This is understandable, since $51(36.7 \%)$ of the anaphylactic deaths in the UK over a 6 year period were due to medication.
Sixteen $(31.4 \%)$ of these deaths resulted from antibiotics, including a 5 -year-old child. ${ }^{3}$ However, only a small proportion of ADRs result from reproducible allergic immunological mechanisms. One meta-analysis found that up to $24 \%$ of inpatient ADRs were characterised as 'allergic and or idiosyncratic' reactions, without requiring further investigation for more detailed determination. ${ }^{4}$ Despite this, prevailing caution has allowed a substantial proportion of children experiencing ADRs to be labelled with 'suspected antibiotic allergy', without further investigation or confirmation.

Identifying and managing suspected antibiotic allergy has now become a clinical imperative, as current practice requires that we have reliable systems in place to mitigate iatrogenic harm and manage risks associated with healthcare interventions. In September 2014, the National Institute of Health and Care Excellence recommended that individuals warrant referral to specialist services if "they are likely to need $\beta$-lactam antibiotics frequently in the future". 5 It can be argued that all children then qualify for investigation as many antibiotic courses may be required over a lifetime, usually in an acute setting. The National Institute of Health and Care Excellence guideline emphasises the need for all healthcare workers to recognise, record and make referrals for suspected antibiotic allergy, while the antibiotic prescription rate among UK general practices is soaring. ${ }^{6}$ The few specialist paediatric allergy services in the UK are widely dispersed and have limited capacity to cope with increased demand for the investigation of suspected drug allergy. ${ }^{7}$

Allergic reactions to antibiotics may be caused by a variety of mechanisms and raise a considerable diagnostic challenge. ${ }^{8}$ The World Health Organisation (WHO) defines ADRs as being either Predictable (type A) or Unpredictable (type B). The Unpredictable type are subclassified into pharmacological drug intolerance, idiosyncratic pharmacodynamic reactions and allergic reactions. Immediate, type 1 hypersensitivity and IgE-mediated drug allergic reactions commonly cause urticaria, angio-oedema and potentially airway and systemic compromise, whereas nonimmediate syndromes may manifest either as localised cutaneous responses, or systemic signs associated with more serious syndromes (figure 1).

Drug provocation tests (DPTs) are recommended as the first-line gold standard investigation among children with mild allergic reactions and rashes to $\beta$-lactams. ${ }^{10}$ However, clinical pathways using patients' histories, skin prick testing (SPT) and intradermal (ID) testing have not been validated against DPT outcome data. Rigorous appraisal of 
Figure 1 Clinical presentation patterns of antibiotic allergy. *Proceed to drug provocation testing only among patients with minor syndrome presentations and when clinical risk benefit favourable. "Avoid drug provocation testing. Caution also with skin testing according to risk-benefit. Further guidance regarding syndrome characteristics available from National Institute for Health and Care Excellence (NICE).

\begin{tabular}{|c|c|c|c|}
\hline & Immediate & Non-immediate & $\begin{array}{l}\text { Non-immediate } \\
\text { suspected serious }\end{array}$ \\
\hline & \multicolumn{3}{|c|}{ Time-scale from first administration } \\
\hline & $<1$ hour & $>1$ hour & $>1$ hour \\
\hline $\begin{array}{l}\text { Possible } \\
\text { associated } \\
\text { symptoms \& } \\
\text { signs }\end{array}$ & $\begin{array}{l}\text { Urticaria, angio-oedema, } \\
\text { airway \& systemic } \\
\text { compromise }\end{array}$ & $\begin{array}{l}\text { Maculopapular } \\
\text { exanthema }\end{array}$ & $\begin{array}{l}\text { Lymph node, joint, mucous } \\
\text { membrane involvement, } \\
\text { bullae, cytopenia, hepatitis, } \\
\text { nephrititis \& vasculitis }\end{array}$ \\
\hline \multirow[t]{5}{*}{$\begin{array}{l}\text { Specific } \\
\text { syndromes } \\
\text { include }\end{array}$} & $\begin{array}{l}\text { * IgE-mediated } \\
\text { anaphylaxis }\end{array}$ & $\begin{array}{l}\text { * Fixed drug } \\
\text { eruption, non- } \\
\text { bullous }\end{array}$ & $\begin{array}{l}\text { \# Drug Rash with Eosinophilia } \\
\text { and Systemic Symptoms }\end{array}$ \\
\hline & $\begin{array}{l}{ }^{*} \text { Non-lgE-mediated } \\
\text { anaphylactoid reactions }\end{array}$ & $\begin{array}{l}\text { * Erythema } \\
\text { multiforme }\end{array}$ & $\begin{array}{l}\text { *Acute Generalised } \\
\text { Exanthematous Pustolosis }\end{array}$ \\
\hline & & & * Serum Sickness Syndrome \\
\hline & & & $\begin{array}{c}\text { * Drug-induced dermatoses } \\
\text { (eg lupus spectrum) }\end{array}$ \\
\hline & & & $\begin{array}{c}\text { \# Stevens Johnson Syndrome \& } \\
\text { \# Toxic Epidermal Necrolysis }\end{array}$ \\
\hline
\end{tabular}

published studies is required to identify accurate, safe and acceptable diagnostic investigations and management strategies, to address this public health concern.

We performed a systematic review of the literature in order to identify best practice principles for diagnosing and managing antibiotic allergy among children and address the following questions: 1. What is the prevalence of antibiotic allergy among children?

2. What are the most accurate clinical investigations for the diagnosis of antibiotic allergy among children, using DPT as the diagnostic gold standard?

3. Do any clinical features of a child's reaction or comorbid risk factors obviate or modify the need for investigation?

4. How long does antibiotic allergy last in children and when should follow-up assessments be planned after diagnosis?

\section{METHODOLOGY}

We systematically searched Medline from inception in 1948 until June 2014. The search strategy combined terms for all major groups of antibiotics through subject headings, and antibiotic syndromes while requiring that children were included (figure 2). Further publications were sourced through hand searches of the literature. No limits were set for language of

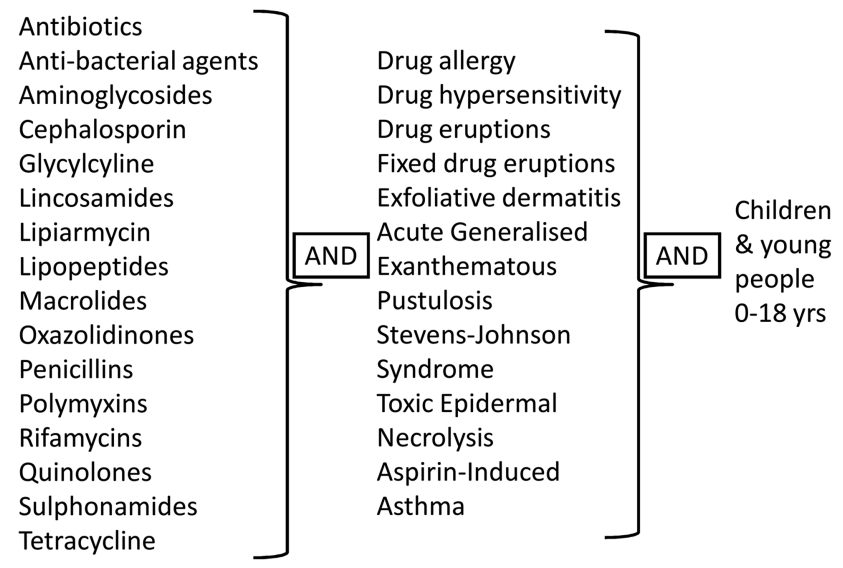

Figure 2 Search strategy. publication, and where articles were not accessible, contact was attempted with authors.

The Medline search retrieved 865 publications and items were selected in accordance with a selection protocol (figure 3 ). ${ }^{11}$ This required that selected publications investigated only children ( $\leq 18$ years of age) or described a specific group thereof within the sample. Reviews, animal models and case reports of less than five subjects were excluded. A hand search was also performed.

Evidence was graded according to a pragmatic score, based closely on the Newcastle-Ottawa Quality Score, as no quality appraisal tool has been published relating to drug allergy evidence. ${ }^{12-14}$ Publications were awarded greater weight of evidence for using DPTs $(+1)$, skin testing $(+1)$, population-based samples $(+1),>99$ subjects $(+1)$, reporting of incomplete testing or follow-up $(+1)$, adjustment for age, sex $(+1)$ and appropriate statistics $(+1)$. Studies of the same quality score were ranked such that larger samples contributed more weight of evidence. Meta-analysis was not appropriate due to heterogeneity in study design. We report studies qualitatively and present individual study data in tables.

\section{RESULTS}

Eight hundred and sixty-five publications were retrieved, with 11 added from hand searches, of which 158 passed screening and resulted in 76 studies being selected for this review.

\section{Question 1: What is the prevalence of antibiotic allergy among children?}

Eleven studies surveyed the prevalence of suspected antibiotic allergy among children and four of these were conducted among the general population, however none used any confirmatory investigations, limiting their value (see online supplementary table S1).

The largest survey used the US National Centre for Health Statistics to trawl 11 years worth of outpatient and accident and emergency department (ED) visit data for ADRs. Of 585932 annual attendances, 253101 (43\%) related to children 0-4 years of age. Antibiotics accounted for $28 \%$ of ADR visits across age groups, with further increases among those of $0-4$ years. ${ }^{15}$ The Swedish Medical Products Agency received 5771 reports of 


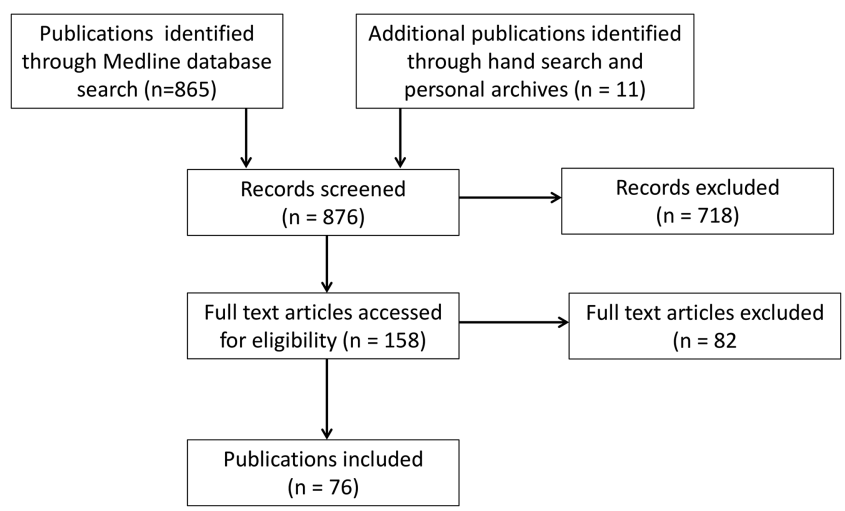

Figure 3 Flow chart of study selection process.

ADRs over 14 years, with 681 (27.2\%) of non-vaccine drug doses implicating antibiotics. ${ }^{16}$ The remaining studies assessing institution records or parental reported histories found that $57-85 \%$ of ADRs were reportedly due to antibiotics.

All studies using investigations to determine antibiotic allergy recruited participants from hospital, raising the risks of selection bias. One study in Switzerland investigated suspected $\beta$-lactam allergy using a DPT-based protocol among consecutive presentations to their ED, and elicited positive reactions among $6.8 \%$ of patients. A Portuguese study investigated likely antibiotic allergic patients among 1426 general paediatric outpatients, who returned questionnaires. Three of the 25 suspected antibiotic allergy subjects had either positive ID or DPTs to index antibiotics ( $\beta$-lactams, co-trimoxazole and macrolides), yielding $12 \%$ prevalence of positive tests among those with likely antibiotic allergy and $0.21 \%$ diagnoses among general paediatric attendees. ${ }^{17}$

The majority of other case series sampled data from allergy department referrals, further raising selection bias. A large case series reporting investigations among 3275 French drug allergy referrals found that children had a significantly lower rate of positive ID or DPT results when compared with adults, especially after maculopapular rashes $(10.6 \%$ positive tests among children vs $16.5 \%$ among adults, $\mathrm{p}<0.0001) .{ }^{18}$

These studies suggest that children aged up to 4 years present most commonly to drug allergy clinics, suggesting that young children may be more susceptible to antibiotic allergy. However, parents of younger children may pay greater attention to adverse reactions and seek more robust medical investigation, leading to bias. Additionally, none of these studies adjusted for how commonly antibiotics were used by the populations investigated.

In summary, the best evidence suggests that $0.21 \%$ of unselected general paediatric outpatients demonstrate positive tests for antibiotic allergy, whereas $6.8 \%$ of children attending ED for suspected $\beta$-lactam allergy develop allergic signs on DPT.

Question 2: What are the most accurate clinical investigations for the diagnosis of antibiotic allergy among children, using DPT as the diagnostic gold standard?

Consensus has established that DPT is the gold standard investigation for drug allergy, since varying mechanisms may be attributed and reproducibility is one of the key diagnostic criteria. ${ }^{19} 20$

Four publications performed DPT to the index antibiotic among all children included in their studies. Two of these also used skin testing among their sample, allowing its performance to be ascertained. However, among 41 (54\%) of the selected studies, positive skin testing was assumed to indicate antibiotic allergy, preventing comparison with the gold standard (see online supplementary tables S2 and S3).
Caubet et al reported the best quality publication comparing skin testing to DPT results for index antibiotics across their sample. The authors consented 88 of 108 consecutive presentations of suspected $\beta$-lactam allergy presenting to their Swiss ED. Each participant underwent skin prick and ID testing, followed by DPT with a $48 \mathrm{~h}$ continuing course. Eleven (13\%) of the 88 patients demonstrated positive ID testing, none reacted to SPT or serum-specific IgE. Six $(6.8 \%)$ demonstrated positive oral DPT with non-serious rashes, one at $30 \mathrm{~min}$ and five producing cutaneous signs between $7 \mathrm{~h}$ and $12 \mathrm{~h}$ later. Only four of the six reacting on DPT had positive ID tests, leaving seven children with false-positive ID results and therefore giving a positive predictive value of only $36.4 \%$. $\beta$-lactam ID testing had a sensitivity of $66.7 \%$, and specificity of $91.5 \%$ with respect to DPT.

One other study performed skin and DPT to clarithromycin after previous suspected reactions among 64 children in Florence, Italy. Mori et al demonstrated that nine (14\%) demonstrated positive ID responses, and yet only four (6\%) resulted in positive DPTs. Urticaria and angio-oedema arose within $20 \mathrm{~min}$ of DPT Clarithromycin dosing for two participants, and delayed maculopapular rashes developed after 3 days in two others. The authors' clarithromycin ID testing protocol demonstrated $75 \%$ sensitivity, $90 \%$ specificity and $33.3 \%$ positive predictive value with respect to DPT.

Two other studies performed DPTs across all subjects to their index antibiotics, supporting the use of DPTs as first-line investigations for antibiotic allergy in children. ${ }^{21} 22$ The same team from Florence investigated consecutive referrals with co-amoxiclav suspension ADRs by performing DPTs to co-amoxiclav itself and sodium benzoate, the suspension preservative. ${ }^{21}$ Eight (9\%) of the 89 consecutive suspected co-amoxiclav allergy referrals demonstrated positive DPT reactions to co-amoxiclav itself, while 10 (11\%) reacted to sodium benzoate and three (3\%) failed both DPTs. Therefore, 21 (24\%) demonstrated a reproducible allergic response to sodium benzoate or co-amoxiclav. A Dutch tem performed DPTs to index agents among 33 children with suspected antibiotic allergy and reported that four $(12 \%)$ produced mild skin reactions after index DPT, confirming reproducible allergy. ${ }^{22} \mathrm{No}$ studies demonstrated that investigation using SPT alone or serum antibiotic antibodies was reliable or useful.

In conclusion, positive predictive values for ID testing to $\beta$-lactam and clarithromycin are very low at $36 \%$ and $33 \%$, respectively. ${ }^{23} 24$ The four studies which reported using DPTs as their principle diagnostic tool resulted in positive signs among $6.3-23.6 \%$ of suspected antibiotic allergy cases. ${ }^{21-24}$ Where DPTs elicited signs, these were usually cutaneous and mild, often arising more than $1 \mathrm{~h}$ post administration (see online supplementary table S2).

\section{Question 3: Do any clinical features of a child's reaction or comorbid risk factors obviate or modify the need for investigation?}

Non-immediate antibiotic allergy syndromes, such as Toxic Epidermal Necrolysis (TEN) and Drug Rash with Eosinophilia and Systemic Symptoms (DRESS), carry significant mortality rates: cautious investigation is warranted (see online supplementary table S3, figure 1). Seven of the nine studies investigating non-immediate suspected serious antibiotic reactions did not investigate cases. One study reported cases reacting to more than one drug of entirely unrelated classes, ${ }^{25}$ suggesting that looking for antibiotic agent-specific causes for these immune responses may be less justified if the problem relates more to constitutional vulnerability or latent virus reactivation. ${ }^{8}$ The two papers conducting ID testing among erythema multiforme and serum 
sickness-like syndrome presentations did not report unsafe adverse effects. $^{26} 27$ Three publications described drug eruption series, with one conducting DPTs to co-trimoxazole among five (14\%) participants without reporting systemic responses. ${ }^{28}$ The remaining studies which assessed non-immediate suspected serious reactions reported likely culprit agents including antibiotics, without reference to their investigation. ${ }^{25} 29-33$

Some studies investigated potential risk factors for immediate antibiotic allergy, however none of these were strong enough to obviate the need for investigation. Three studies highlighted that anaphylaxis, urticaria and angio-oedema index responses were associated with a higher likelihood of positive DPT or ID responses when compared with non-specific rashes, however did not preclude investigation. ${ }^{24} 34-36$ One study suggested that food allergy was a risk factor for $\beta$-lactam allergy among 161 Portuguese children $(p=0.047) .{ }^{37}$ Kidon and See ${ }^{38}$ found that having asthma predisposed towards failing drug DPTs, however was unable to correct for their increased medication requirement.

Overall, it is prudent to take a cautious approach among children describing multiple signs which are consistent with an allergic reaction to antibiotics. If the first dose of an antibiotic course induced immediate anaphylaxis with breathing difficulty or airway signs, or patients fulfil criteria for non-immediate serious syndromes, DPTs may be contraindicated (figures 1 and 4).

\section{Question 4: How long does antibiotic allergy last in children and when should follow-up assessments be planned after diagnosis?}

No single study has followed the natural history of DPT-proven antibiotic allergy with subsequent investigation.

Indeed most interest has focused on finding out whether negative investigation results remain consistently negative. One Israeli study repeated ID testing and DPTs among 98 children up to 5 months after their first investigations were negative. One subject demonstrated a positive response to penicillin ID testing the second time around, and another developed a maculopapular rash $30 \mathrm{~min}$ after the single dose DPT, resulting in two further diagnoses (2\%). ${ }^{39}$ The second American study conducted ID testing 1 month after initial skin and DPT investigations were negative, reporting that $26(14 \%)$ then tested ID positive. ${ }^{40}$
Neither study assessed whether their postinvestigation prevalence was higher than those among healthy control children.

\section{DISCUSSION}

We have performed the first systematic review to appraise evidence for the diagnosis and management of antibiotic allergy in children.

Younger children present more commonly with ADRs, with $43-61 \%$ of episodes originating in 0-4-year-olds. ${ }^{15} 16$ The likely prevalence of positive skin testing and DPTs to antibiotics among general paediatric outpatients is around $0.21 \%,{ }^{17}$ whereas DPT-proven reproducibility among children with suspected antibiotic allergy ranges between $6.3 \%$ and $24 \% .^{21} 2324$ The conduct of DPTs among children with non-serious reactions was safe. Indeed the majority produced delayed cutaneous reactions which are of questionable clinical significance and need not preclude antibiotic usage in a medical emergency. We recommend that suspected non-serious antibiotic allergy should be primarily investigated using DPT-based clinical protocols.

Despite DPT-based diagnosis becoming increasingly common throughout the UK, our literature review finds only four (6\%) papers that performed DPTs to subjects' index antibiotic across all participants with mild reaction histories. No rigorous evidence supports skin and in vitro diagnostic testing; two studies compared ID testing with DPT data across participants. These demonstrated a sensitivity of $66.7 \%$ and $75 \%$, with positive predictive values of $36 \%$ and $33 \%$ for ID testing to $\beta$-lactam and clarithromycin, respectively. ${ }^{23} 24$ These data raise the question of whether skin testing should be undertaken to investigate antibiotic allergy among children at all.

We ensured that a wide range of literature was retrieved using broad search terms and not limiting according to language. Nonetheless, not all publications were retrievable, even after attempting contact with study authors.

Heterogeneity in study design and investigation protocols prevented meta-analysis and assessment of publication bias. The majority of hospital-based case series were vulnerable to selection bias, although better quality publications highlighted prospective introduction of protocols to reduce this (see online supplementary table S2). Nonetheless, since the first publication in 1964, an
Figure 4 Evidence-led approach to the diagnosis and management of antibiotic allergy in children.
Focused clinical history regarding illness, antibiotic exposure and following symptoms with examination, as guided by NICE recommendations

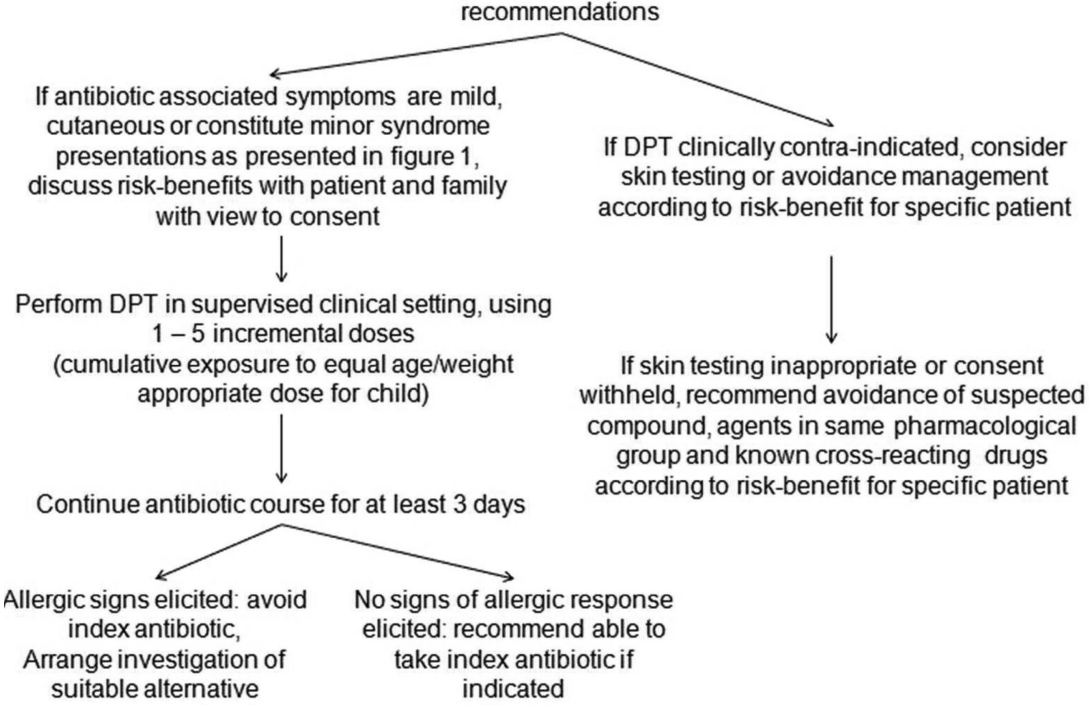


Figure 5 Benefits and drawbacks of investigating allergy to antibiotics using drug provocation testing.

\begin{tabular}{|c|c|}
\hline Benefits & Drawbacks \\
\hline Age and dose appropriate protocols & $\begin{array}{l}\text { Risk of administering systemic doses } \\
\text { (asthma must be well controlled) }\end{array}$ \\
\hline Acceptable for most paediatric patients & Difficult to interpret subjective symptoms \\
\hline Good safety record & DPT to only one agent possible over a few days \\
\hline Negative responses are painless & $\begin{array}{l}\text { Should not be undertaken whilst taking } \\
\text { antihistamines }\end{array}$ \\
\hline Low risk of false positive diagnosis & $\begin{array}{l}\text { Low risk of false negative diagnostic outcomes as } \\
\text { co-factors typically absent at time of DPT }\end{array}$ \\
\hline \multirow[t]{4}{*}{$\begin{array}{c}\text { Antibiotic course may be extended for late } \\
\text { reactions }\end{array}$} & $\begin{array}{l}\text { If positive, subsequent DPT required to identify } \\
\text { safe alternatives }\end{array}$ \\
\hline & $\begin{array}{l}\text { Mild symptoms and signs may develop on } \\
\text { subsequent doses }\end{array}$ \\
\hline & $\begin{array}{l}\text { Requires supervised clinical setting; once validated } \\
\text { protocols may allow secondary and primary care }\end{array}$ \\
\hline & Low risk of re-sensitisation to antibiotic with DPT \\
\hline
\end{tabular}

increased variety of antibiotics has become available and laboratory techniques for detecting sensitisation have changed enormously. ${ }^{41}$ This may explain considerable changes in prevalence data detected over time. ${ }^{4142}$ Where outlined, some DPT protocols did not require ongoing course completion to ascertain delayed reactions. We were unable to construct a receiver operating characteristic curve analysis to compare investigations, as too few study designs would have been eligible for inclusion therein.

\section{Context of findings}

Early consensus guidelines for investigating antibiotic allergy proposed that positive SPT and ID results fulfilled diagnostic criteria for antibiotic allergy, based on two early case series which sporadically used DPT. ${ }^{43-45}$ It has since become clear that skin testing is of limited accuracy with $8.4-13.7 \%$ of ID negative adults demonstrating symptoms on DPT. ${ }^{46}$

Additionally, in clinical practice, we determine the patient's status towards the suspected antibiotic and towards suitable alternatives. Therefore, the majority of ID testing panels include a range of reagents at varying concentrations. ${ }^{47}$ This is unacceptable to a large proportion of paediatric patients, particularly since their discomfort can prevent adequate investigation. There appears to be little reason to continue to use ID testing to antibiotics among children.

We recommend that suspected non-serious antibiotic allergy should be primarily investigated using DPT-based clinical protocols, as has become routine among large centres in the UK and abroad (figure 4). Incremental DPTs should be undertaken with expert clinical supervision for the first cumulative dose, to ensure appropriate surveillance of symptoms and signs, and excellent management of allergic reactions (figure 5). ${ }^{19}$ The index antibiotic preparation should be used where possible, to best support positive diagnosis of an allergic syndrome. ${ }^{19-21}$ Intravenous DPT may be undertaken only where paediatric intensive care facilities are available. A 3 day course of the suspected antibiotic should be continued after negative DPTs, to allow elicitation of non-immediate responses and reduce concomitant bacterial resistance. ${ }^{24}$ Delayed cutaneous reactions that are mild and last for less than $24 \mathrm{~h}$ may not be clinically concerning (with the exception of erythema multiforme and the suspected serious syndromes listed in figure 1) and may not preclude administration of the same antibiotic should there be sufficient clinical indication.

Although DPTs are the clinical gold standard and are safe among well children, they still have some limitations. For example, DPT results may still have the capacity to produce false-negative results. ${ }^{34}{ }^{39}$ Unlike common food allergens, antibiotic molecules are typically low molecular weight and haptenisation may be required to facilitate immune activation. There may be many cofactors that facilitate this process; for example, studies investigating food challenges have highlighted that intercurrent illnesses, poor control of comorbid atopic disease and exposure to other drugs may reduce the threshold at which patients demonstrate allergic responses. ${ }^{48}$ Necessarily, the majority of children who experience ADRs to antibiotics are unwell when they develop suspected allergic responses. There is currently no evidence investigating what proportion of children passing their DPT may later experience an allergic recurrence at the time of future illness. These factors should not be recreated when preparing for a DPT to test reproducibility as this may compromise safety. ${ }^{49}$

DPT-based protocols also require that only one antibiotic is investigated for several days at a time, increasing the time taken for each investigation. Positive DPT responses will typically require that a second DPT be undertaken during another visit to identify a suitable alternative antibiotic, with consequences for resource allocation.

As we move towards adopting DPT-based diagnosis for children with suspected antibiotic allergy, it is imperative for us to collate high quality data regarding children undergoing DPTs, their conduct and safety. There is also a need to design carefully controlled multicentre follow-up studies to ascertain their longterm validity. Cost-benefit analyses associated with DPT-based challenge regimens are also indicated, given the prevalence of suspected antibiotic allergy and the scarce resources available for allergy services. The safety of DPT-based protocols require robust investigation before it would become appropriate to consider advocating this practice more widely, with the aim to improve accessibility to the appropriate investigation of antibiotic allergy in children. 
In the interim, we recommend that suspected non-serious antibiotic allergy should be primarily investigated using DPT-based clinical protocols in tertiary drug allergy centres.

Contributors TM and GdT conceived the four premise questions. TM performed the systematic review and drafted the article. ATF and GL provided feedback. All authors designed the pragmatic management recommendations.

Competing interests GdT was on the National Institute for Clinical Excellence Guideline Group for "Drug Allergy: diagnosis and management of drug allergy in adults, children and young people" published September 2014. GdT joint led and wrote the Royal College of Paediatrics and Child Health (RCPCH) Care Pathway for Children with Drug Allergies, ADC 2011. GdT currently leads the Paediatric Drug Allergy service which conducts challenges and skin testing at the Children's Allergies Department, St Thomas' Hospital, Guy's and St Thomas' NHS Foundation Trust. GL has received research funding from ALK Abello and sponsorship from Novartis, Sodilac and Nestle.

Provenance and peer review Commissioned; externally peer reviewed.

\section{REFERENCES}

1 Macy E. Elective penicillin skin testing and amoxicillin challenge: effect on outpatient antibiotic use, cost, and clinical outcomes. J Allergy Clin Immunol 1998; 102:281-5.

2 Tsakok T, du Toit G, Flohr C. Pediatric urticaria. Immunol Allergy Clin North Am 2014;34:117-39.

3 Pumphrey RS. Lessons for management of anaphylaxis from a study of fatal reactions. Clin Exp Allergy 2000;30:1144-50.

4 Lazarou J, Pomeranz BH, Corey PN. Incidence of adverse drug reactions in hospitalized patients: a meta-analysis of prospective studies. JAMA 1998;279:1200-5.

5 NICE Drug Allergy Clinical Guideline Group. Drug allergy: diagnosis and management of drug allergy in adults, children and young people. September 2014. Accessed from http://www.nice.org.uk/guidance/CG183 on 5th October 2104.

6 Hawker JI, Smith S, Smith GE, et al. Trends in antibiotic prescribing in primary care for clinical syndromes subject to national recommendations to reduce antibiotic resistance, UK 1995-2011: analysis of a large database of primary care consultations. J Antimicrob Chemother 2014:69:3423-30.

7 Joint Royal College of Physicians and Royal College of Pathologists Working party. Allergy Services: Still not meeting the unmet need. 2010.

8 Caubet JC, Pichler WJ, Eigenmann PA. Educational case series: mechanisms of drug allergy. Pediatr Allergy Immunol 2011;22:559-67.

9 Romano A, Caubet JC. Antibiotic allergies in children and adults: from clinical symptoms to skin testing diagnosis. J Allergy Clin Immunol Pract 2014;2:3-12.

10 du Toit G, Lloyd K, Sinnott L, et al. The RCPCH care pathway for children with drug allergies: an evidence and consensus based national approach. Arch Dis Child 2011;96(Suppl 2):i15-18.

11 Moher D, Liberati A, Tetzlaff J, et al. Preferred reporting items for systematic reviews and meta-analyses: the PRISMA statement. BMJ 2009;339:b2535.

12 Marrs T, Bruce KD, Logan $\mathrm{K}$, et al. Is there an association between microbial exposure and food allergy? A systematic review. Pediatr Allergy Immunol 2013;24:311-20.

13 Wells GA, Shea B, O'Connell D, et al. The Newcastle-Ottawa Scale (NOS) for assessing the quality of nonrandomised studies in meta-analyses 2014; 19:476-88

14 Flohr C, Pascoe D, Williams HC. Atopic dermatitis and the 'hygiene hypothesis': too clean to be true? Br J Dermatol 2005;152:202-16.

15 Bourgeois FT, Shannon MW, Valim C, et al. Adverse drug events in the outpatient setting: an 11-year national analysis. Pharmacoepidemiol Drug Saf 2010;19:901-10.

16 Kimland $\mathrm{E}$, Rane $\mathrm{A}$, Ufer $\mathrm{M}$, et al. Paediatric adverse drug reactions reported in Sweden from 1987 to 2001. Pharmacoepidemiol Drug Saf 2005; 14:493-9.

17 Rebelo GE, Fonseca J, Araujo L, et al. Drug allergy claims in children: from self-reporting to confirmed diagnosis. Clin Exp Allergy 2008;38:191-8.

18 Rubio M, Bousquet PJ, Gomes E, et al. Results of drug hypersensitivity evaluations in a large group of children and adults. Clin Exp Allergy 2012;42:123-30.

19 Aberer W, Bircher A, Romano A, et al. Drug provocation testing in the diagnosis of drug hypersensitivity reactions: general considerations. Allergy 2003; 58:854-63.

20 Torres MJ, Mayorga C, Leyva L, et al. Controlled administration of penicillin to patients with a positive history but negative skin and specific serum IgE tests. Clin Exp Allergy 2002;32:270-6.
21 Mori F, Barni S, Pucci N, et al. Cutaneous adverse reactions to amoxicillin-clavulanic acid suspension in children: the role of sodium benzoate. Curr Drug Saf 2012;7:87-91.

22 Mattheij $M$, de Vries $E$. A suspicion of antibiotic allergy in children is often incorrect. J Allergy Clin Immunol 2012;129:583-4.

23 Mori F, Barni S, Pucci N, et al. Sensitivity and specificity of skin tests in the diagnosis of clarithromycin allergy. Ann Allergy Asthma Immunol 2010;104:417-9.

24 Caubet JC, Kaiser L, Lemaitre B, et al. The role of penicillin in benign skin rashes in childhood: a prospective study based on drug rechallenge. J Allergy Clin Immunol 2011;127:218-22.

25 Dore J, Salisbury RE. Morbidity and mortality of mucocutaneous diseases in the pediatric population at a tertiary care center. J Burn Care Res 2007;28:865-70.

26 Chopra R, Roberts J, Warrington RJ. Severe delayed-onset hypersensitivity reactions to amoxicillin in children. CMAJ 1989;140:921-3.

27 Blanca-Lopez N, Zapatero L, Alonso E, et al. Skin testing and drug provocation in the diagnosis of nonimmediate reactions to aminopenicillins in children. Allergy 2009;64:229-33.

28 Morelli JG, Tay YK, Rogers M, et al. Fixed drug eruptions in children. J Pediatr 1999;134:365-7.

29 Ferrandiz-Pulido C, Garcia-Fernandez D, Dominguez-Sampedro P, et al. Stevens-Johnson syndrome and toxic epidermal necrolysis in children: a review of the experience with paediatric patients in a university hospital. J Eur Acad Dermatol Venereol 2011:25:1153-9.

30 Forman R, Koren G, Shear NH. Erythema multiforme, Stevens-Johnson syndrome and toxic epidermal necrolysis in children: a review of 10 years' experience. Drug Safety 2002;25:965-72.

31 Ginsburg CM. Stevens-Johnson syndrome in children. Pediatr Infect Dis 1982;1:155-8.

32 King BA, Geelhoed GC. Adverse skin and joint reactions associated with oral antibiotics in children: the role of cefaclor in serum sickness-like reactions. J Paediatr Child Health 2003:39:677-81.

33 Raucci U, Rossi R, Da Cas R, et al. Stevens-johnson syndrome associated with drugs and vaccines in children: a case-control study. PLOS ONE 2013;8:e68231.

34 Graff-Lonnevig V, Hedlin G, Lindfors A. Penicillin allergy-a rare paediatric condition? Arch Dis Child 1988;63:1342-6.

35 Ponvert C, Le Clainche L, de Blic J, et al. Allergy to beta-lactam antibiotics in children. Pediatrics 1999;104:e45.

36 Ponvert C, Perrin Y, Bados-Albiero A, et al. Allergy to betalactam antibiotics in children: results of a 20-year study based on clinical history, skin and challenge tests. Pediatr Allergy Immunol 2011;22:411-18

37 Chambel M, Martins P, Silva I, et al. Drug provocation tests to betalactam antibiotics: experience in a paediatric setting. Allergol Immunopathol (Madr) 2010;38:300-6.

38 Kidon MI, See Y. Adverse drug reactions in Singaporean children. Singapore Med J 2004:45:574-7.

39 Hershkovich J, Broides A, Kirjner L, et al. Beta lactam allergy and resensitization in children with suspected beta lactam allergy. Clin Exp Allergy 2009:39:726-30.

40 Pichichero ME, Pichichero DM. Diagnosis of penicillin, amoxicillin, and cephalosporin allergy: reliability of examination assessed by skin testing and oral challenge. J Pediatr 1998;132:137-43.

41 Jost BC, Wedner HJ, Bloomberg GR. Elective penicillin skin testing in a pediatric outpatient setting. Ann Allergy Asthma Immunol 2006;97:807-12.

42 Perez-Rodriguez E, Martin-Conde L, Sanchez-Machin I, et al. Beta-lactam allergy in children. Pediatr Allergy Immunol 2006;17:236-7.

43 Torres MJ, Blanca M, Fernandez J, et al. Diagnosis of immediate allergic reactions to beta-lactam antibiotics. Allergy 2003;58:961-72.

44 Macy E, Burchette RJ. Oral antibiotic adverse reactions after penicillin skin testing: multi-year follow-up. Allergy 2002;57:1151-8.

45 Sogn DD, Evans RIII, Shepherd GM, et al. Results of the National Institute of Allergy and Infectious Diseases Collaborative Clinical Trial to test the predictive value of skin testing with major and minor penicillin derivatives in hospitalized adults. Arch Intern Med 1992;152:1025-32.

46 Messaad D, Sahla H, Benahmed S, et al. Drug provocation tests in patients with a history suggesting an immediate drug hypersensitivity reaction. Ann Intern Med 2004;140:1001-6.

47 Brockow K, Garvey LH, Aberer W, et al. Skin test concentrations for systemically administered drugs -- an ENDA/EAACI Drug Allergy Interest Group position paper. Allergy 2013;68:702-12.

48 Summers CW, Pumphrey RS, Woods CN, et al. Factors predicting anaphylaxis to peanuts and tree nuts in patients referred to a specialist center. J Allergy Clin Immunol 2008;121:632-8.

49 Bierman CW, Van Arsdel PP Jr. Penicillin allergy in children: the role of immunological tests in its diagnosis. J Allergy 1969;43:267-72. 